\title{
COMPORTAMENTO ALIMENTAR EIDOSOS: UM ESTUDO SOBRE A ESTÉTICA E A GASTRONOMIA
}

\author{
Jennifer Lacerda ${ }^{1}$ \\ Angélica Bezerra Gomes² \\ Isabelle Patriciá Freitas Soares Chariglione ${ }^{3}$ \\ Gislane Ferreira de $\mathrm{Melo}^{4}$ \\ Marileusa Dosolina Chiarello ${ }^{5}$
}

resumo

O objetivo desta pesquisa foi avaliar a percepção de diferentes preparações gastronômicas entre idosos. Participaram desta pesquisa 38 idosos (idade $=67,65, \mathrm{DP}= \pm 6,15$ ), que degustaram uma preparação montada de duas formas, uma mais e outra menos estética. Esses idosos responderam a um questionário semiestruturado para os dados sociodemográficos, além de medidas hedônicas relacionadas às preparações e medidas psicológicas para avaliação dos estados de humor. Para análise dos dados, foram utilizados os testes Shapiro-Wilks, Wilcoxon, Mann Whitney e Kruskal-Wallis. Além disso, optou-se por trabalhar com o delta da diferença com três grupos, de acordo com a sua preferência de

1 Graduada em Gastronomia. Mestre em Gerontologia pela Universidade Católica de Brasília. Gastrônoma. E-mail: jeangelo.lacerda@gmail.com.

2 Graduanda em Psicologia pela Universidade Católica de Brasília. E-mail: angelicagomesabg@ gmail.com.

3 Graduada em Psicologia. Doutora em Cognição e Neurociências pela Universidade Católica de Brasília. Psicóloga. E-mail: isabelle.chariglione@p.ucb.br.

4 Graduada em Educação Física. Pós-Doutorado em Educação Física pela Universidade Católica de Brasília. Profissional de Educação Física. E-mail: gislane.melo@gmail.com.

5 Graduada em Farmácia. Pós-Doutorado em Processos Biotecnológicos pela Universidade Católica de Brasília. Farmacêutica e Bioquímica. E-mail: marileusa.chiarello@gmail.com. 
prato, utilizando o teste Qui-Quadrado (Monte Carlo). Os resultados demonstraram que o prato mais estético foi melhor avaliado por todos os idosos ( $p=0,000)$, que o prato mais estético foi percebido como maior quantidade de sal quando dividido por faixa etária ( $p=0,020)$, não havendo diferença entre idosos e idosas $(p \geq 0,05)$. Também não foram encontradas diferenças quanto às percepções pelo fato de o idoso cozinhar ou não, tampouco pela presença de ansiedade e depressão, visto que o grupo tinha baixos escores dessas. Assim, a utilização de pratos mais estéticos na gastronomia pode vir a favorecer a melhora do comportamento alimentar neste grupo, assim como melhorar a percepção de gostos, especialmente do salgado, que é diminuído no processo de envelhecer.

palavras-chave

Gastronomia. Estética. Idoso. Percepção Visual.

\section{Introdução}

A gastronomia é definida por Brillat-Savarin (1841) como o conhecimento de tudo o que se refere ao homem na medida em que ele se alimenta. Para Braune e Franco (2017), essa ciência é como o artesanato, já que exige de quem a faz habilidade, conhecimento e técnica. A partir de fatores como preparação da comida, arrumação do prato e decoração, é possível transformar a refeição em um momento especial, criando um elo com outros prazeres da vida (CHIACHIRI FILHO, 2008).

O comportamento alimentar está diretamente relacionado à trajetória pessoal do sujeito, pois as influências que determinam o comportamento alimentar de um indivíduo não ocorrem somente no jovem e no adulto, iniciam na infância, quando a alimentação da criança passa a ser igual à da família, sendo estimulada pela cultura na qual está inserido. Essas preferências alimentares então podem ser construídas de acordo com a sociedade, cultura, crenças e religião de cada povo. Sendo assim, para um melhor entendimento do comportamento alimentar dos indivíduos, é necessário conhecer um pouco e considerar os diferentes usos e costumes socioculturais (VAZ; BENNEMANN, 2014).

Ao alimentar-se, todos os cinco sentidos são aguçados, então estimulá-los nesse momento pode proporcionar satisfação sensorial (NASCIMENTO, 2007). O sabor de um alimento, para Monteiro (2009), não é uma característica somente 
dele, mas uma interação entre ele e quem o consome. Nesse sentido, questões biopsicossociais e histórico-culturais impactam na percepção gustativa de um alimento para cada pessoa. Apesar de o sujeito ter a necessidade biológica de alimentar-se, há quesitos psicológicos e sociais que o farão ter um comportamento alimentar específico, pois o paladar pode ser aperfeiçoado por lembranças e modelos de identificação que são construídos culturalmente (NASCIMENTO, 2007; BRILLAT-SAVARIN, 1841).

Pimentel (2009) relata sobre os sentidos que estão relacionados ao sabor. A audição, estimulada pelos sons emitidos na mastigação, exerce influência no sabor quando as texturas são sentidas - crocância, maciez, dureza. $\mathrm{O}$ aparato físico da boca é capaz de capturar texturas, temperaturas e consistências, complementando o sentido da visão. O cheiro pode causar inúmeras reações no organismo, sendo determinante para permitir ou não o que será ingerido. A visão é de especial importância, pois "se come primeiro com os olhos", e é onde o paladar se associa a uma boa apresentação dos alimentos (VEIROS; PROENÇA, 2003). Os aspectos sensoriais também devem ser adequados qualitativamente para atrair atenção e seduzir o comensal, transformando o momento das refeições em um momento de prazer. Para que isso seja possível aos sujeitos, é necessário que todos os sentidos estejam em bom funcionamento (OLIVEIRA, 2010).

Indivíduos com mais de 60 anos, considerados idosos no Brasil, por vezes podem não usufruir de todo esse prazer, pois, a partir dessa idade, é possível que já existam alguns declínios sensoriais, como olfato e paladar, que são afetados e acabam por interferir no apetite (BUENO et al., 2008; MOREIRA, 2014). A perda de gemas gustativas das papilas da língua pode diminuir a um terço comparado a um indivíduo jovem, dessa forma, a sensibilidade por gostos primários como doce, salgado, azedo é diminuída. Isso, combinado ao uso de fármacos, comum na velhice (DOUGLASS; HECKMAN, 2010), pode alterar ainda mais o sabor, o cheiro e a salivação, levando-os a mudar seus hábitos alimentares. Assim, se faz importante pensar em estratégias para propiciar um melhor momento para as refeições, onde a inapetência, as carências nutricionais e, por vezes, o excesso de condimentos artificiais, sal, gordura e açúcares podem ser diminuídos por uma melhor apresentação, aqui denominada estética.

A estética, originada do grego aisthésis, tem sua raiz aisth no verbo aisthanomai, que significa "sentir com o coração", usando, assim, sensações e percepções. Essa foi uma das definições defendidas por Platão na Grécia Antiga em discussões com outros filósofos, como Aristóteles e Pitágoras (STRATI, 2007). 
Para Oliveira (2010), a culinária, além de uma dimensão biológica, tem sua dimensão estética no que se refere aos sabores, cores, sons e cheiros e o que essas sensações podem provocar em quem degusta um prato. Para Braune e Franco (2017), definir a gastronomia tão e somente pelo fato de o homem ter a necessidade de se alimentar não está completa, pois o homem é um animal estético. Então, a gastronomia nasce quando o alimento é preparado com arte, a partir do que antes alimentaria apenas o corpo, transformando-se em experiência estética e em prazer de alimentar a alma. O comensal que prova um prato feito com arte analisa que sua textura, temperatura, aroma e sabor estão conectados de forma harmoniosa, experimentando o belo, usando mais de um sentido.

É importante ressaltar também que os transtornos de humor, como depressão e ansiedade, são desordens psiquiátricas que alteram o humor por semanas ou meses, alterando funções como sono, libido e apetite (VISMARI; ALVES; PALERMO-NETO, 2008). Nesse sentido, é primordial a investigação e prevenção desses transtornos para um melhor comportamento alimentar dos idosos.

Diante do exposto, este estudo tem o objetivo de avaliar a percepção de diferentes preparações gastronômicas entre idosos, buscando unir os estudos de gastronomia e estética para um melhor momento nas refeições, tanto nos sentidos perceptivos quanto nutricionais.

\section{Método}

O presente estudo é do tipo quantitativo, quase-experimental e transversal, conforme definições de Sampieri, Collado e Lucio (2013). Esta pesquisa e o seu referido Termo de Consentimento Livre e Esclarecido foram submetidos ao sistema do Comitê de Ética em Pesquisa, e aprovados por meio do protocolo CAAE n. ${ }^{\circ} 74773217.3 .0000 .0029$.

Participaram desta pesquisa 38 idosos, com média de idade igual a 67,65 anos ( $\mathrm{DP}= \pm 6,15)$, dos 40 inscritos e selecionados por uma amostra de conveniência. Foi critério de inclusão ter idade igual ou superior a 60 anos. Os critérios de exclusão foram: ter menos de 60 anos ou ageusia (perda do sentido do paladar), ser deficiente visual, ter incapacidade motora de manipular os utensílios de cozinha para a degustação das preparações, ter alergia à lactose, ser fumante ou ainda estar gripado no dia da coleta.

O estudo foi realizado na Universidade Católica de Brasília, Distrito Federal, Brasil, mais especificamente no Laboratório de Análise Sensorial, 
localizado no Laboratório de Gastronomia. Esse laboratório é destinado aos estudos de análise sensorial, sendo composto de uma antessala equipada com uma mesa grande e cadeiras e por seis cabines separadas, onde os participantes não têm contato entre eles durante os testes de análise sensorial.

Neste estudo, foram selecionados materiais para as preparações gastronômicas e para as avaliações dos idosos conforme descritos a seguir. Para as preparações gastronômicas, utilizou-se a Ficha Técnica de Preparação (FTP), instrumento contendo descrição dos ingredientes, quantidades, cortes empregados e modo de preparo. A FTP é usada para manter a padronização das preparações em diferentes dias ou quantidade de vezes de preparo. Para Fonseca (2000), a FTP é um dos documentos mais importantes de um restaurante, pois registra todo o processo de elaboração de um prato, as matérias-primas utilizadas e suas quantidades, assim como seus custos. Serve também para padronizar a montagem dos pratos através de fotos, manter um histórico das preparações do restaurante, permitir a comparação de informações de consumo e facilitar nas compras, além de manter o mesmo aspecto sensorial e físico (AKUTSU et al., 2005).

Os alimentos utilizados para a preparação gastronômica (farofa de cuscuz de milho) foram sêmola de milho, tomate, cebola, ervas frescas, ovo, manteiga, carne de sol cozida e sal. Essa preparação foi escolhida pela facilidade de ser servida em temperatura ambiente, ser de fácil e rápida execução e por ter versatilidade quanto à montagem.

As preparações foram confeccionadas no Laboratório de Gastronomia, equipado com bancadas, fogões, balanças digitais, geladeiras, freezers, fornos, pias para lavagem de mãos e louças, ou seja, todos os utensílios e equipamentos de cozinha necessários para a execução dos pratos. Os pratos seguiram duas apresentações estéticas (mais estética e menos estética) conforme definição de Oliveira (2010), que entende que a manipulação de diferentes níveis de sabores, cores, sons ou cheiros podem provocar diferentes percepções em quem degusta um prato.

Destaca-se aqui que nas duas versões, a mais estética e a menos estética, a quantidade servida era a mesma, os ingredientes eram os mesmos, o sabor era o mesmo, controlados conforme todas as informações supracitadas. Os pratos apresentados diferenciaram apenas na forma como eram montados e na louça onde foram servidos.

Para as avaliações das percepções dos idosos em relação às preparações, utilizou-se um questionário semiestruturado na seleção dos sujeitos da pesquisa, sendo constituído de 15 perguntas, avaliado por pares da área da Gerontologia e aplicado previamente em um piloto para a adequação do 
instrumento, conforme Coelho (2018). Esse instrumento auxiliou a pesquisadora a selecionar os participantes pelos critérios de inclusão e exclusão e caracterizou a amostra quanto a idade, sexo, grau de escolaridade, estado civil e informações para contato.

Também foi empregada a escala hedônica, para avaliar a percepção do sabor. É um instrumento que mede o grau de satisfação em relação a um determinado produto. O sujeito da pesquisa marca o grau de gostar e desgostar de algo através de avaliações que são convertidas em pontos. Possui um ponto central de diferença e, por isso, é sempre descrita em número ímpar de três a nove.

Utilizou-se, ainda, a Escala de Ansiedade de Beck, que consiste em instrumento de autorrelato utilizado para medir aspectos da ansiedade, dos quais foram selecionados os itens que passaram a compor o inventário. Este é composto por 21 itens que refletem, somaticamente, afetivamente e cognitivamente, os sintomas característicos de ansiedade. $\mathrm{O}$ instrumento foi validado em 2001 por Cunha, e desde que foi traduzido vem sendo utilizado em grupos psiquiátricos e não psiquiátricos (CUNHA, 2001).

Outro instrumento de avaliação utilizado foi a Escala de Depressão de Beck, que autoavalia sintomas depressivos, desenvolvido com base em observações clínicas por Aron Beck, em 1961. Esse instrumento, validado e traduzido no Brasil em 1982, é composto por 21 itens que descrevem manifestações comportamentais cognitivas, afetivas e somáticas da depressão. Cada categoria contém quatro ou cinco alternativas e a pontuação para cada categoria varia de zero a três, desde a ausência de sintomas depressivos até a presença de sintomas intensos (BECK; STEER, 1990).

Os procedimentos para a execução da pesquisa seguiram quatro etapas, definidas como: a) recrutamento dos idosos, b) capacitação dos alunos, c) avaliação psicológica dos idosos e d) avaliação gastronômica dos idosos. A etapa de recrutamento dos idosos durou cerca de 30 dias e esses foram selecionados por meio de divulgação no e-mail institucional da Universidade Católica de Brasília, convidando os funcionários que tivessem mais de 60 anos e/ou seus familiares. Além do e-mail institucional, também foram utilizadas as mídias sociais. Os idosos interessados deveriam entrar em contato com a pesquisadora por e-mail.

A etapa da capacitação dos estudantes de Psicologia e Gastronomia deu-se em grupo e teve duração de 2 horas, sendo conduzida pela pesquisadora e sua orientadora. Nessa capacitação, foram abordadas questões éticas em pesquisas aplicadas com seres humanos e orientações sobre a aplicação dos instrumentos. 
A etapa da avaliação psicológica dos idosos durou uma semana, e cada idoso tinha um horário pré-agendado dentro desse período, com tempo médio de avaliação de 30 minutos. Essa avaliação era individual e realizada com o auxílio de um estudante de Psicologia.

Após essa primeira avaliação, cada idoso, também individualmente, foi dirigido às cabines de análise sensorial para a realização da etapa de avaliação gastronômica. Nessa etapa, os idosos experimentaram as preparações e preencheram um formulário denominado escala hedônica para verificar suas percepções acerca do que foi degustado. Os idosos eram acompanhados individualmente por estudantes de Gastronomia, e levavam um tempo médio de 10 minutos para degustação e análise de cada prato.

Em síntese, os idosos foram avaliados durante cinco dias consecutivos, com preparações novas feitas a cada dia por uma profissional da Gastronomia, durante os quais provaram uma das amostras mais estética (Figura 1) e menos estética (Figura 2). A cada dia, a ordem das amostras era modificada para que não se criassem vieses que pudessem dar tendência de primazia (preferência pelo primeiro prato) ou recência (preferência pelo último prato) a ser degustado.

Figura 1 - Prato mais estético.

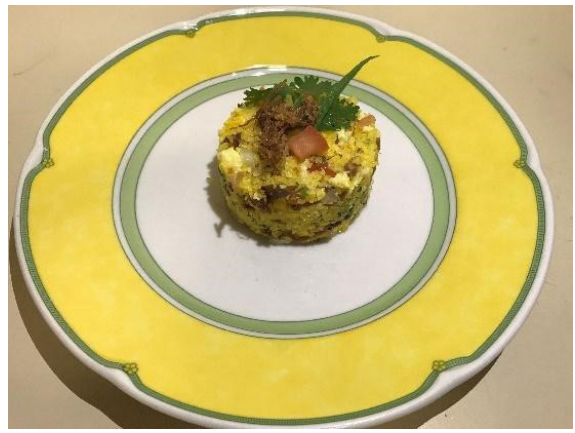

Fonte: Elaborada pelas autoras.
Figura 2 - Prato menos estético.

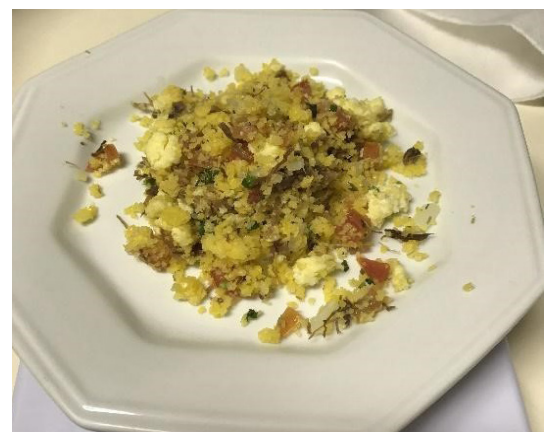

Fonte: Elaborada pelas autoras.

Os idosos eram sempre divididos em dois horários, 15h e 16h. O horário foi pensado para que os idosos não apresentassem um apetite exacerbado nem sua ausência, pois tais fatores poderiam influenciar no resultado, sendo recomendado que esse tipo de avaliação seja realizado cerca de duas horas antes ou depois das refeições (TEIXEIRA, 2009).

Para análise dos dados, foram utilizadas estatísticas descritivas que permitiram resumir, descrever e compreender os dados por meio das médias, 
desvios e frequências. Inicialmente, avaliou-se a normalidade dos dados (Shapiro-Wilks), tanto por grupos (idade, sexo, estado de humor e gostar de cozinhar) quanto de forma geral. Não foram observadas não normalidades em nenhuma das análises. Assim, utilizou-se o teste de Wilcoxon (comparação entre prato mais e menos estético), bem como o teste de Mann Whitney (comparação dos níveis de ansiedade) e Kruskal-Wallis (comparação dos níveis de depressão e faixas etárias). Optou-se por trabalhar com o delta da diferença, formando grupos nos quais os idosos preferiram o prato mais estético (P1), o prato menos estético (P2) e um grupo (P3) que não viu diferença entre os dois pratos. Assim, utilizou-se o teste Qui-Quadrado (Monte Carlo) para a comparação de frequências entre sexo, gostar de cozinhar, estados de humor e faixa etária. O nível de significância estipulado foi de $\mathrm{p} \leq 0,05$. As análises foram realizadas por meio do software SPSS-IBM 22.0, devidamente registrado.

\section{Resultados}

A média de idade da amostra foi de 67,65 ( $\mathrm{DP}= \pm 6,15$ ), sendo a idade mínima igual a 60 anos e a máxima igual a 87 anos. Esta pesquisa é composta, como na maioria dos estudos, por uma amostra majoritariamente feminina $(n=28)$ e indivíduos com estado civil de casado(a) $(n=20)$. Em relação ao grau de escolaridade, houve o predomínio de sujeitos com ensino médio completo $(\mathrm{n}=11)$ e com nível superior completo $(\mathrm{n}=11)$, conforme Tabela 1.

Tabela 1 - Dados descritivos da amostra.

\begin{tabular}{llll}
\hline Variável & Nomes & $\mathrm{n}$ & $\%$ \\
\hline Sexo & Feminino & 28 & 73,7 \\
& Masculino & 10 & 26,3 \\
& Total & 38 & 100 \\
Estado Civil & Casado(a) & 20 & 52,6 \\
& Divorciado(a) & 5 & 13,2 \\
& Solteiro(a) & 3 & 7,9 \\
& Viúvo(a) & 10 & 26,3 \\
Escolaridade & Total & 38 & 100 \\
& Fundamental Incompleto & 6 & 15,8 \\
& Fundamental Completo & 6 & 15,8 \\
& Ensino Médio Incompleto & 2 & 5,3 \\
& Ensino Médio Completo & 10 & 26,3
\end{tabular}




\begin{tabular}{llll}
\hline Variável & Nomes & $\mathrm{n}$ & $\%$ \\
\hline \multirow{4}{*}{ Medicamentos } & Superior Incompleto & 3 & 7,9 \\
& Superior Completo & 11 & 28,9 \\
& Total & 38 & 100 \\
& Sim & 33 & 86,8 \\
& Não & 5 & 13,2 \\
& Total & 38 & 100 \\
\hline
\end{tabular}

Fonte: Elaborada pelas autoras.

Para avaliar as variáveis visual, combinação de cores, cheiro, textura, sabor e quantidade de sal, nos pratos 1 (mais estético) e 2 (menos estético), utilizou-se o teste de Wilcoxon, com variáveis em estudo métricas, escalonadas de 1 a 9, e uma variação entre Desgostei Extremamente (1) e Gostei Extremamente (9). Pode-se perceber que apenas um item teve diferença significativa entre todos os participantes (homens e mulheres avaliados conjuntamente), sendo este o visual, como apresentado na Tabela 2.

Tabela 2 - Comparação dos pratos mais e menos estéticos avaliados por todos os idosos.

\begin{tabular}{llllll}
\hline \multirow{2}{*}{ Variáveis } & \multicolumn{2}{l}{ Prato 1-mais estético } & \multicolumn{2}{c}{ Prato $2-$ menos estético } & p \\
& Média & DP & Média & DP & \\
\hline Visual & 8,62 & 0,63 & 7,21 & 2,12 & $0,000^{*}$ \\
Combinações de cores & 8,36 & 0,93 & 8,05 & 1,29 & 0,11 \\
Cheiro & 7,92 & 1,61 & 7,92 & 1,32 & 0,74 \\
Textura & 8,03 & 1,34 & 8,0 & 1,31 & 1,00 \\
Sabor & 8,31 & 1,23 & 8,23 & 1,30 & 0,71 \\
Quantidade de sal & 8,28 & 1,25 & 8,33 & 1,03 & 0,97 \\
\hline
\end{tabular}

Fonte: Elaborada pelas autoras.

DP: Desvio Padrão. * Teste Qui-Quadrado.

Ao comparar a percepção entre idosos do sexo masculino e feminino sobre as variáveis supracitadas, visual dos pratos, combinação de cores, cheiro, textura, sabor e quantidade de sal, após a aplicação do Qui-Quadrado (Monte Carlo), verificou-se que as percepções não são diferentes entre homens e mulheres. Analisou-se, também, por meio do Qui-Quadrado (Monte Carlo), se gostar de 
cozinhar exercia influência nas variáveis investigadas nos dois tipos de prato, e também não foram observadas diferenças significativas.

Para uma análise mais minuciosa dos dados, os idosos foram divididos em três diferentes faixas de idade, sendo: G1: 60 a 64 anos (12); G2: 65 a 69 anos (15); e G3: 70 anos ou acima (11), para uma análise da distinção de percepção de cores e pratos de preferência entre as diferentes faixas etárias. Percebeu-se que, de forma geral, não apresentam diferenças significativas em suas percepções. No entanto, ao se falar na combinação de cores, os grupos responderam de forma distinta: G2 e G3 não perceberam diferenças quanto a esse quesito no prato mais estético e menos estético, já G1 avaliou o prato mais estético com uma melhor combinação de cores, com $p=0,063$ na associação linear, ou seja, com $\mathrm{p}$ aproximando-se ao valor significante de referência de ( $\mathrm{p} \leq 0,05)$, conforme Tabela 3 .

Tabela 3 - Comparação entre faixas etárias dos idosos em percepção de cores e pratos de preferência.

\begin{tabular}{llllll}
\hline & & $\mathrm{P} 1$ & $\mathrm{P} 2$ & $\mathrm{P} 3$ & Total \\
\hline \multirow{4}{*}{$\begin{array}{llll}\text { Grupo 1 } \\
\text { (60 a 64 anos) }\end{array}$} & Quantidade de indivíduos & 6 & 2 & 4 & 12 \\
& \% dentro do grupo & $50,0 \%$ & $16,7 \%$ & $33,3 \%$ & $100,0 \%$ \\
& \% do total & $46,2 \%$ & $33,3 \%$ & $21,1 \%$ & $31,6 \%$ \\
& Quantidade de indivíduos & 6 & 1 & 8 & 15 \\
Grupo 2 & \% dentro do grupo & $40,0 \%$ & $6,7 \%$ & $53,3 \%$ & $100,0 \%$ \\
(65 a 69 anos) & \% em relação à percepção de cores & $46,2 \%$ & $16,7 \%$ & $42,1 \%$ & $39,5 \%$ \\
& \% do total & $15,8 \%$ & $2,6 \%$ & $21,1 \%$ & $39,5 \%$ \\
Grupo 3 & Quantidade de indivíduos & 1 & 3 & 7 & 11 \\
(acima de 70 & \% dentro do grupo & $9,1 \%$ & $27,3 \%$ & $63,6 \%$ & $100,0 \%$ \\
anos) & \% do total & $7,7 \%$ & $50,0 \%$ & $36,8 \%$ & $28,9 \%$ \\
& 2,6\% & $7,9 \%$ & $18,4 \%$ & $28,9 \%$ \\
\hline
\end{tabular}

Fonte: Elaborada pelas autoras.

P1: Grupo de idosos que preferiram o prato mais estético. P2: Grupo de idosos que preferiram o prato menos estético. P3: Grupo de idosos que não observaram a diferença entre os dois pratos. Linear-by-linear $p=0,063$ (houve tendência à diferença significativa).

Observou-se também diferença no fator sabor, em específico, a quantidade de sal, com $\mathrm{p}=0,02$, na associação linear. Quatro sujeitos em todo o estudo perceberam mais a quantidade de sal no prato mais estético, dentre eles, três (75\%) estão no grupo G3, de acordo com a Tabela 4. 
Tabela 4 - Comparação entre faixas etárias dos idosos na percepção da quantidade de sal e pratos de preferência.

\begin{tabular}{llllll}
\hline & & $\mathrm{P} 1$ & $\mathrm{P} 2$ & $\mathrm{P} 3$ & Total \\
\hline \multirow{4}{*}{$\begin{array}{llll}\text { Grupo 1 } \\
\text { (60 a 64 anos) }\end{array}$} & Quantidade de indivíduos & 0 & 0 & 12 & 12 \\
& \% dentro do grupo & $0,0 \%$ & $0,0 \%$ & $100,0 \%$ & $100,0 \%$ \\
& \% do totação à percepção de sal & $0,0 \%$ & $0,0 \%$ & $38,7 \%$ & $38,7 \%$ \\
& Quantidade de indivíduos & 1 & 2 & 12 & 15 \\
Grupo 2 & \% dentro do grupo & $6,7 \%$ & $13,3 \%$ & $80,0 \%$ & $100,0 \%$ \\
(65 a 69 anos) & \% em relação à percepção de sal & $25,0 \%$ & $66,7 \%$ & $38,7 \%$ & $39,5 \%$ \\
& \% do total & $2,6 \%$ & $5,3 \%$ & $31,6 \%$ & $39,5 \%$ \\
Grupo 3 & Quantidade de indivíduos & 3 & 1 & 7 & 11 \\
(acima de 70 & \% dentro do grupo & $27,3 \%$ & $9,1 \%$ & $63,6 \%$ & $100,0 \%$ \\
anos) & \% em relação à percepção de sal & $75,0 \%$ & $33,3 \%$ & $22,6 \%$ & $28,9 \%$ \\
& \% do total & $7,9 \%$ & $2,6 \%$ & $18,4 \%$ & $28,9 \%$ \\
\hline
\end{tabular}

Fonte: Elaborada pelas autoras.

P1: Grupo de idosos que preferiram o prato mais estético. P2: Grupo de idosos que preferiram o prato menos estético. P3: Grupo de idosos que não observaram a diferença entre os dois pratos.

Linear-by-linear $p=0,020$ (houve diferença significativa).

Ao avaliar se os estados de humor poderiam influenciar nas percepções sensoriais dos idosos, observou-se que a amostra estudada apresentou inicialmente apenas níveis rebaixados quanto aos níveis de ansiedade (100\% entre os níveis leve e mínimo) e depressão (86,8\% entre os níveis leve e mínimo), conforme Escala Beck de Ansiedade e Depressão. Utilizando-se os testes de Mann Whitney (Ansiedade) e Kruskal-Wallis (Depressão) também se verificou que os sujeitos respondiam de forma semelhante, independentemente dos níveis de ansiedade e depressão, ou seja, o estado de humor, nesses níveis, não alterou a percepção dos idosos.

\section{Discussão}

Na presente pesquisa, a discussão será realizada por estudos feitos em amostras compostas por adultos, por não se ter encontrado, até o presente momento, estudos que avaliem intervenções na gastronomia e na estética com a população idosa, o que mais uma vez destaca a importância de se realizar mais pesquisas sobre o assunto e a possibilidade deste trabalho vir a contribuir para a construção de novos saberes e maiores conexões entre a Gastronomia e a Gerontologia. 
Através do presente estudo, pôde ser observado que a estética interferiu na percepção visual dos idosos em ambos os sexos e em todas as faixas etárias. Em relação às percepções entre homens e mulheres, verificou-se que ambos perceberam, de forma similar, o que vai de acordo com Ribeiro (2014), que, em seu estudo sobre consumo de vinhos em Portugal, observou-se que as mulheres, ao contrário do que se pensava, não valorizavam o design da garrafa de vinho em escala maior que os homens. Ambos os sexos se importaram com o visual do recipiente do vinho, corroborando que homens e mulheres se comportam de forma semelhante quando se trata da parte estética.

O resultado dessas pesquisas não condiz com os estudos de Bourdieu (1984), que identificou diferenças entre homens e mulheres no que diz respeito aos gostos e comportamentos quando se alimentam; ou com os resultados de Hyde e Feller (1981 apud MOJET; CHRIST-HAZELHOF; HEIDEMA, 2001), em que os homens, quando comparados às mulheres, perceberam maior intensidade de amargura no café e na quinoa; ou com a pesquisa de Simchen et al. (2006), ao apontar que mulheres percebem mais o cheiro e o sabor do que os homens.

No que se refere à textura, isto é, a sensação sentida ao mastigar, os resultados da pesquisa atual foram divergentes dos estudos de Delgado et al. (2006), em que os homens deram maior pontuação à textura macia da carne experimentada do que as mulheres. Tais resultados foram contrários ao entendimento de Oliveira-Pinto (2014), ao mostrar o dimorfismo no bulbo olfatório humano, indicando que as mulheres têm mais neurônios e células gliais que os homens nas regiões cerebrais responsáveis por essas funções.

Neumann, Schauren e Adami (2016), em estudo sobre palatabilidade na presença de sal usando solução com cloreto de sódio, não verificaram diferenças perceptuais entre os sexos, assim como nesta pesquisa. Porém, na comparação entre as faixas etárias em relação à quantidade de sal, a percepção do grupo G3 (idosos com mais de 70 anos) surpreendeu positivamente, pois alguns idosos avaliaram melhor o prato mais estético em relação a sua quantidade de sal, como pôde ser visto na Tabela 4. A associação linear revelou significância, $p=0,02$, demonstrando uma tendência do grupo em sentir maior presença de sal no prato mais estético, diferente do esperado, visto que, nessa faixa etária, as gemas gustativas podem diminuir até um terço comparado a um jovem, então há alterações gustativas que dificultam a percepção do gosto salgado (BUENO et al., 2008; MOREIRA, 2014). Nesse sentido, pode-se inferir que o fator que interferiu nessa percepção foi a estética, sugerindo que pratos mais elaborados esteticamente podem ser percebidos como mais saborosos, por serem melhor percebidos quanto à quantidade de sal. 
Ainda comparando as faixas etárias, os idosos perceberam de forma distinta as combinações de cores nos pratos 1 (mais estético) e 2 (menos estético). Nesse sentido, os idosos do G1, de 60 a 64 anos, perceberam que o prato mais estético tinha uma maior e/ou melhor combinação de cores do que o menos estético. Em síntese, tal constatação indica que, para esses idosos, a forma como as cores estão distribuídas no prato, ou seja, a decoração deste, interfere na avaliação que eles dão ao prato. Essa percepção condiz com os estudos de Menegazzo et al. (2011), que afirmam a importância de uma variedade de cores no prato, facilitando a ingestão das preparações por ser mais atrativo e possibilitando o consumo de uma maior diversidade de minerais e vitaminas.

Apesar de não estar dentro dos objetivos específicos do estudo, é importante destacar que o baixo nível de escolaridade não influenciou de forma negativa a percepção do prato mais estético, como percebido em estudos anteriores. Pelo contrário, a preferência pelo prato mais estético foi geral, contrariando o que argumenta Bourdieu (2007) ao dizer que o julgamento estético é eminentemente social e que tem base na classe social e no nível de educação. A diversidade de escolaridade do grupo estudado comprovou que não apenas pessoas com mais anos de estudo e que conhecem a gastronomia preferem o prato mais estético, o que vai de acordo com as afirmações de que todas as classes sociais se importam com a forma como a comida é servida e que isso lhes traz prazer, como foi abordado por Certeau, Giard e Mayol (2008). E que, como afirma Oliveira (2010), o prazer de comer os belos pratos gastronômicos e mesmo os mais simples, como a comida caseira do cotidiano, pode ser aproveitado por todos.

Deve-se, então, levar em consideração que o sabor de um alimento não é uma característica somente dele, mas uma interação entre ele e quem o consome, e que o paladar pode ser aperfeiçoado por lembranças e modelos de identificação e é uma construção de cada cultura, gerada através dos tempos, envolvendo ingredientes e o modo como são preparados (FREYRE, 2002; MONTEIRO, 2009; NASCIMENTO, 2007; BRILLAT-SAVARIN, 1841).

\section{Conclusão}

Através da realização da presente pesquisa, constata-se que o prato mais estético foi mais bem avaliado visualmente por toda a amostra estudada, o que demonstra a necessidade de investimento em pratos mais elaborados esteticamente para idosos, culminando em um maior prazer na alimentação, já que "comemos primeiro com os olhos". Neste estudo, o visual, a combinação 
de cores e a quantidade de sal foram percebidos de formas distintas de acordo com a estética do prato - mais estético e menos estético - e entre as faixas etárias dos idosos. Refere-se a um aspecto positivo, na medida em que comprova que os idosos estudados estavam adequados biologicamente com suas percepções gustativas. Porém, sugere-se que mais estudos nesse sentido sejam realizados, com uma amostra maior, para que haja a verificação ou não de tendências a uma percepção distinta entre eles, já que elas apareceram nos dados aqui apresentados.

Em relação à combinação de cores, os idosos de 60 a 64 anos compreenderam que o prato mais estético tinha uma melhor combinação de cores. Já em relação à quantidade de sal, alguns indivíduos com mais de 70 anos perceberam o prato mais estético como o mais salgado. Esse dado se faz importante, pois, levando em consideração que biologicamente os idosos perdem cada vez mais o paladar para os gostos básicos, infere-se que essa percepção está ligada à estética do prato, fazendo com que o gosto salgado seja percebido de maneira mais acentuada. Dessa forma, a estética de uma preparação gastronômica pode sim influenciar na percepção do seu sabor, respondendo a uma das hipóteses deste estudo.

Na pesquisa em questão, idosos que cozinhavam não perceberam alterações nos sabores, texturas, combinação de cores e quantidade de sal de acordo com os pratos oferecidos. Além disso, verificou-se que os sujeitos da coleta de dados responderam de forma semelhante independentemente do sexo e dos níveis de ansiedade e depressão, ou seja, o estado de humor, nesses níveis, não alterou a percepção dos idosos.

Pode-se perceber que ainda há algumas lacunas em relação à alimentação dos idosos e como a estética pode influenciar em uma alimentação mais prazerosa. Apesar de neste estudo ter ficado claro que o prato mais estético seria o de melhor preferência para os idosos, inclusive estes percebendo uma maior quantidade de sal por uma manipulação exclusivamente estética, pede-se parcimônia e mais estudos para a generalização dessa conclusão. Então, sugere-se que haja mais estudos que abordem esse assunto, com a possibilidade de aprofundamento em questões como percepção e cultura, história de vida, percepções de acordo com o sexo, faixas etárias e meio social e suas influências. 


\section{EATING HABITS AND THE ELDERLY: A STUDY ON AESTHETICS AND GASTRONOMY}

The objective of this research was to evaluate the perception of different gastronomic preparations among the elderly. Thirty-eight elderly (age $=67.65, \mathrm{SD}= \pm 6.15$ ) participated in this study, who tasted a preparation assembled in two ways, one more and one less aesthetic. Those elderly filled in a semi-structured questionnaire for sociodemographic data, as well as hedonic measures related to the preparation and psychological measures to assess mood states. For data analysis, the Shapiro-Wilks, Wilcoxon, Mann Whitney and Kruskal-Wallis tests were used. In addition, we chose to work with the difference delta with three groups, according to their plate preference, using the Chi-square test (Monte Carlo). The results showed that the most aesthetic dish was better evaluated by all the elderly $(p=0.000)$, that the more aesthetic dish was perceived as having a greater amount of salt when divided by age group ( $p=0.020)$, and that there was no difference between elderly men and elderly women ( $p \geq 0.05)$. Also, no differences on perceptions were found regarding the fact of whether the elderly cooked or not, as well as whether they had anxiety and depression, since the group had low anxiety and depression scores. Thus, the use of more aesthetic dishes in the gastronomy may favor the improvement of the food behavior in this group, as well as improve the perception of tastes, especially the salty one, that is diminished in the aging process.

keywords

Gastronomy. Aesthetics. Elderly. Visual Perception.

referências

AKUTSU, Rita de Cássia et al. A ficha técnica de preparação como instrumento de qualidade na produção de refeições. Revista de Nutrição, Campinas, v. 18, n. 2 , p. 277-279, 2005.

BECK, Aaron; STEER, Robert. Manual for the Beck Anxiety Inventory. San Antonio: Psychological Corporation, 1990.

BOURDIEU, Pierre. Distinction: a social critique of the judgement of taste. Cambridge: Harvard University Press, 1984.

A distinção: crítica social do julgamento. São Paulo: Edusp; Porto Alegre: 

2017.

BRILLAT-SAVARIN, Jean Anthelme. Physiologie du goût. Paris: Charpentier, 1841.

BUENO, Júlia Macedo et al. Avaliação nutricional e prevalência de doenças crônicas não transmissíveis em idosos pertencentes a um programa assistencial. Ciência \& Saúde Coletiva, Rio de Janeiro, v. 13, n. 4, p. 1237-1246, 2008.

CERTEAU, Michel de; GIARD, Luce; MAYOL, Pierre (Org.). A invenção do cotidiano 2: morar, cozinhar. 8. ed. Petrópolis: Vozes, 2008.

CHIACHIRI FILHO, Antônio Roberto. O sabor das imagens. 2008. 144 f. Tese (Doutorado em Comunicação e Semiótica)-Pontifícia Universidade Católica de São Paulo, São Paulo, 2008.

COELHO, Jennifer Ângelo de Lacerda. A influência da gastronomia e da estética na percepção sensorial de idosos. 2018. 67 f. Dissertação (Programa Stricto Sensu em Gerontologia)-Universidade Católica de Brasília, Brasília, 2018.

CUNHA, Jurema Alcides. Manual da versão em português das Escalas Beck. São Paulo: Casa do Psicólogo, 2001.

DELGADO, Eduardo Francisquine et al. Brazilian consumers' perception of tenderness of beef steaks classified by shear force and taste. Scientia Agrícola, Piracicaba, v. 63, n. 3, p. 232-239, 2006.

DOUGLASS, Rebecca; HECKMAN, George. Drug-related taste disturbance: a contributing factor in geriatric syndromes. Canadian Family Physician, Mississauga, v. 56, n. 11, p. 1142-1147, 2010.

FONSECA, Marcelo Traldi. Tecnologias gerenciais de restaurantes. São Paulo: Editora Senac, 2000.

FREYRE, Gillberto. Açúcar. São Paulo: Companhia das Letras, 2002.

MENEGAZZO, Manoela et al. Avaliação qualitativa das preparações do cardápio de centros de educação infantil. Revista Nutrição, Campinas, v. 24, n. 2, p. 243-251, 2011.

MOJET, Jos; CHRIST-HAZELHOF, Elly; HEIDEMA, Johannes. Taste perception with age: generic or specific losses in threshold sensitivity to the five basic tastes? Chem Senses, Oxford, v. 26, n. 7, p. 845-860, 2001.

MONTEIRO, Marlene. Percepção sensorial dos alimentos em idosos. Revista Espaço para a Saúde, Londrina, v. 10, n. 2, p. 34-42, 2009.

MOREIRA, Morvan Melo. O envelhecimento da população brasileira: intensidade, feminização e dependência. Revista Brasileira de Estudos de População, Brasília, v. 15, n. 1, p. 79-94, 2014.

NASCIMENTO, Angelina de Aragão Bulcão. Comida: prazeres, gozos e transgressões. Salvador: EDUFBA, 2007

NEUMANN, Luisa; SCHAUREN, Bianca Coletti; ADAMI, Fernanda Sherer. Sensibilidade gustativa de adultos e idosos. Revista Brasileira de Geriatria e Gerontologia, Rio de Janeiro, v. 19, n. 5, p. 797-808, 2016.

OLIVEIRA, Sandra Ramalho e. Sentidos à mesa: saberes além dos sabores. São Paulo: Rosari, 2010.

OLIVEIRA-PINTO, Ana et al. Sexual dimorphism in the human olfactory bulb: females have more neurons and glial cells than males. PLOS ONE, San Francisco, v. 9, n. 11, p. e111733, 2014. Disponível em: <https://journals.plos.org/plosone/article?id=10.1371/ journal.pone.0111733>. Acesso em: 30 maio 2018. 
PIMENTEL, Márcia Ulhôa. Considerações acerca da importância da inclusão da dimensão estética em produto final de um buffet direcionado para clientes classe " $A$ " $e^{\text {" }} B$ ". 2009. 36 f. Monografia (Especialização em Gestão de Hospitalidade)-Universidade de Brasília, Brasília, 2009.

RIBEIRO, Sara Inês Diogo. O comportamento dos consumidores de vinho em Portugal: impacto do género. 2014. 52 f. Dissertação (Mestrado em Marketing)-Instituto Superior de Economia e Gestão, Lisboa, 2014.

SAMPIERI, Roberto Hernández; COLLADO, Carlos Fernánez; LUCIO, María del Pilar. Metodologia de pesquisa. 5. ed. Porto Alegre: Penso, 2013.

SIMCHEN, U. et al. Odour and taste sensitivity is associated with body weight and extent of misreporting of body weight. European Journal of Clinical Nutrition, Berlin, v. 60, n. 6, p. 698-705, 2006.

STRATI, Antonio. Organização e estética. Rio de Janeiro: FGV, 2007.

TEIXEIRA, Lilian Viana. Análise sensorial na indústria de alimentos. Revista do Instituto de Laticínios Cândido Tostes, Juiz de Fora, v. 64, n. 366, p. 12-21, 2009

VAZ, Diana Souza; BENNEMANN, Rose Mari. Comportamento alimentar e hábito alimentar: uma revisão. Revista UNINGÁ Review, v. 20, n. 1, p. 108-112, out./dez. 2014

VEIROS, Marcela Boro; PROENÇA, Rossana. Avaliação qualitativa das preparações do cardápio em uma unidade de alimentação e nutrição - método AQPC. Nutrição em Pauta, Campo Belo, v. 11, n. 62, p. 36-42, 2003.

VISMARI, Luciana; ALVES, Glaucie Jussilane; PALERMO-NETO, João. Depressão, antidepressivos e sistema imune: um novo olhar sobre um velho problema. Revista de Psiquiatria Clínica, São Paulo, v. 35, n. 5, p. 196-204, 2008.

Data de Submissão: 30/05/2018

Data de Aprovação: 21/10/2018 
